\title{
Patterns of white matter degeneration in remote brain areas from the basal ganglion lesion of ischemic stroke patients with motor impairment
}

\section{xuejin cao}

Southeast University https://orcid.org/0000-0002-3684-8092

\section{Zan Wang}

Southeast University, Zhongda hosiptial

\section{Xiaohui Chen}

Southeast University Zhongda Hospital

Yanli Liu

Southeast University Zhongda Hospital

\section{Wei Wang}

Southeast University Zhongda Hospital

\section{Idriss Ali Abdoulaye}

Southeast University

Xi Yang

Southeast University Zhongda Hospital

Shiyao Zhang

Southeast University

\section{Xiaoying Guo}

Southeast University

\section{Shanshan Wu}

Southeast University

Yijing Guo ( $\sim$ guoyijingseu@126.com )

Southeast University https://orcid.org/0000-0002-6638-8963

\section{Research article}

Keywords: Ischemic stroke, Diffusion tensor imaging, Structural network, Multivariate pattern analysis, Network-based statistic

Posted Date: November 11th, 2020

DOI: https://doi.org/10.21203/rs.3.rs-36124/v3 
License: (c) (i) This work is licensed under a Creative Commons Attribution 4.0 International License. Read Full License 


\section{Abstract}

Background: Diffusion tensor imaging (DTI) studies have revealed distinct white matter characteristics of the brain following diseases. Beyond the lesion-symptom mapping, recent studies have demonstrated extensive structural and functional alterations of remote areas to local lesions caused by stroke in the brain. Here, we further investigated the structural changes from a global level using DTI data through multivariate pattern analysis (MVPA) and network-based statistic (NBS).

Methods: Ten ischemic stroke patients with basal ganglia lesions and motor dysfunctions and eleven demographically matched adults as controls underwent brain Magnetic Resonance Imaging scans. DTI data were processed to obtain fractional anisotropy (FA) maps and MVPA was used to explore brain regions that play an important role in classification based on FA maps. The white matter (WM) structural network was constructed by the deterministic fiber tracking approach according to the Automated Anatomical Labeling (AAL) atlas. NBS was used to explore differences in structural networks between groups.

Results: MVPA applied to FA images correctly identified stroke patients with a statistically significant accuracy of $100 \%$ ( $P \leq 0.001)$. Compared with the controls, the study patients showed FA reductions in the perilesional basal ganglia and brainstem, with a few showing reductions in bilateral frontal lobes. Using NBS, we found a significant decrease in FA-weighted WM subnetwork in stroke patients.

Conclusions: We identified some patterns of WM degeneration affecting brain areas remote to the ischemic lesion, revealing the abnormal organization of WM network in stroke patients, which may be helpful for the understanding of the neural mechanisms of stroke sequela.

\section{Background}

Focal brain lesions could affect the overall performance of brain networks. In the past decades, numerous neuroimaging studies have investigated the structural and functional reorganizations after stroke [1]. Diffusion tensor imaging (DTI) is commonly used to detect the structural integrity of the white matter. Previous DTI researches in stroke patients calculated several diffusion tensor indicators (e.g. fractional anisotropy (FA), mean diffusivity (MD), radial diffusivity (RD) ) in various regions along the corticospinal tract (CST) of the lesioned hemisphere and contralateral hemisphere [2,3], or computed the CST integrity [4,5]. These imaging indicators were later correlated to function outcomes, further establishing them as prediction markers. It has been discovered that certain correlations between changes in specific fiber bundles and functional outcomes, such as aphasia [6], neglect [7], paralysis [8], etc. Besides the CST command of motor functions, the involvement of other fibers and brain areas in movement control is not well understood. Therefore, neural degeneration in some regions might cause specific functional manifestations. We made a pattern recognition classification of FA maps of the brain in an attempt to explore regions that are closely related to motor function. 
Multivariate pattern analysis (MVPA) is a method of imaging data analysis based on machine learning and pattern recognition. It involves the use of pattern classification algorithms to extract spatial patterns from neuroimaging data for individual characteristics analysis [9]. MVPA has the advantage of considering interregional correlations and searching for abnormalities throughout the entire brain because it adopts an unbiased and whole-brain method without the region of interest (ROI) being artificially set [10]. This method has been widely used in psychiatry related studies to analyze mild changes that can be observed in subclinical populations [11,12].

White matter structural connectivity can be modeled as a network. A network-based statistics (NBS) method is to control the family-wise error rate during mass univariate testing of every connection of the network. NBS is also performed to investigate the interregional correlations of the brain on a global level $[13,14]$. We utilized these methods to explore subtle relevant changes and abnormalities of structural networks of the brain for a further understanding of the intrinsic brain structural basis of ischemic stroke patients with residual motor dysfunctions.

\section{Methods}

\section{Participants}

Ten right-handed stroke patients (mean age $56.7 \pm 10.5$ years) from the Southeast University affiliated Zhongda Hospital were recruited for this study from March 2019 to December 2019. The inclusion criteria for patients were as follows:(1) age $\geq 20$ and age $\leq 80$ years $\rrbracket(2)$ first-onset of ischemic stroke with the involvement of the basal ganglia; (3) pure motor deficits; (4) stable conditions after treatment of acute stroke without recurrence. The exclusion factors: (1) a history of neurological or psychiatric disorders, prior or subsequent symptomatic stroke; (2) brain abnormalities unrelated to the infarct lesions; (3) MRI contraindications. All of the affected extremities were evaluated for motor function. The motor outcome of the affected limbs was evaluated by the Fugl-Meyer assessment (FMA), including the upper and lower extremities [15]. Also, The Brunnstrom stage (BRS) was adopted. Recovery of the affected extremities was scored on a 6-point scale ( $1=$ severe; $6=$ normal) [16]. The clinical characteristics of the stroke patients are summarized in Table 2. Eleven demographically matched healthy controls (mean age $61.5 \pm 7.8$ years) were also recruited.

\section{Image Acquisition}

Diffusion Tensor Images were acquired using a 3.0-Tesla Philips (Ingenia) Medical Systems equipped with a Synergy-L Sensitivity Encoding (SENSE) head coil, using a single echo planar imaging (EPI) sequence, 33 diffusion-weighted images $\left(b=1000 \mathrm{~s} / \mathrm{mm}^{2}\right)$ and a reference T2-weighted image with no diffusion weighting $\left(b=0 \mathrm{~s} / \mathrm{mm}^{2}\right)$ were obtained with the following acquisition parameters: voxel size $=2 \times 2 \times 2 \mathrm{~mm}^{3}$, gap $=0 \mathrm{~mm}$;echo time $(\mathrm{TE})=107 \mathrm{~ms}$; repetition time $(\mathrm{TR})=5835 \mathrm{~ms}$; field of view $(\mathrm{FOV})=$ $256 \times 256 \mathrm{~mm}^{2} ;$ flip angle $(\mathrm{FA})=90^{\circ} ;$ matrix $=128 \times 128$; slices $=75$. 
High-resolution T1-weighted axial images covering the whole brain were obtained by a 3D-magnetization prepared rapid gradient-echo (MP-RAGE) sequence: $\mathrm{TR}=9.6 \mathrm{~ms} ; \mathrm{TE}=3.7 \mathrm{~ms} ; \mathrm{FA}=9^{\circ}$; matrix $=256 \times 256$; FOV $=256 \times 256 \mathrm{~mm}^{2} ;$ voxel size $=1 \times 1 \times 1 \mathrm{~mm}^{3} ;$ gap $=0 \mathrm{~mm}$, number of slices $=140$. Additionally, sagittal fluid attenuated inversion recovery (FLAIR) images were obtained with the following parameters: $\mathrm{TE}=110 \mathrm{~ms}, \mathrm{TR}=7000 \mathrm{~ms}$, Inversion time $(\mathrm{TI})=2200 \mathrm{~ms}, \mathrm{FA}=90^{\circ}$, matrix size $=480 \times 480, \mathrm{FOV}=250 \times$ $250 \mathrm{~mm}^{2}$, slice thickness $=5 \mathrm{~mm}$, slices $=20$.

\section{Lesion mapping}

Lesion-side normalization was performed to place lesions on the left side of the brain. For the patients with lesions in the right hemisphere, the images were flipped from the right to the left along the midsagittal line to simplify the comparison with the other patients with stroke. According to previous investigators, lesion masks of each patient were manually segmented on individual structural MRI images (T1-weighted MP-RAGE and FLAIR images) using MRIcron software (http://www.mricro.com). After the spatial normalization of all individual lesion masks, a lesion overlap image for all patients was constructed (Fig. 1) [17,18,19].

\section{DTI Data Pre-Processing and Network Definition}

DTI data analysis was performed by a pipeline toolbox for analyzing brain diffusion images (PANDA, http://www.nitrc.org/projects/panda) [20].The main procedure includes: (1) correcting for the head motion and eddy current effects using the FMRIB's Diffusion Toolbox (FDT); (2) calculating the diffusion tensor (DT) metric---FA for each voxel using the DTIFIT tool; (3) normalizing: registrations of all the individual FA images to the FMRIB58_FA template by calling the FNIRT tool [21]. The FA maps were obtained and the FA values of bilateral CST were computed. Whole-brain tractography was performed for each subject in the native diffusion space based on fiber assignment by continuous tracking (FACT) algorithm [22]. All voxels with fractional anisotropy (FA) $\geq 0.2$ were used as seed points; the FA and curvature thresholds of path tracing were set to 0.2 and $45^{\circ}$, respectively.

FA weighted networks were constructed from 116 nodes defined according to the Automated Anatomical Labeling (AAL) atlas [23]. The entire cerebral cortex was automatically partitioned into 116 anatomical regions of interest (ROIs) (45 ROls for each cerebral hemisphere, 26 ROIs for the cerebellum) using the AAL algorithm. The weight of the edges in the network was defined as the mean FA value of the connected fibers between each pair nodes. In order to reduce false-positive connections, two nodes were considered structurally connected only when at least 3 fibers were reconstructed between them [24].

\section{Multivariate pattern analysis}

Pattern classification analysis could be used to examine the differences in the FA values between groups. A specific MVPA approach known as support vector machine (SVM) was implemented using PRoNTo (Pattern Recognition for Neuroimaging toolbox) software (http://www.mlnl.cs. ucl.ac.uk/pronto/) [25]. Individual FA maps were treated as points located in a high dimensional space. A linear decision 
boundary in this high dimensional space was defined by a hyperplane that separated the individual brain images according to a class label (i.e., patients vs. controls)(Fig. 2A). A more detailed description of the SVM can be found in the previous reports [11]. The receiver operating characteristic curve (ROC), sensitivity and specificity of the FA classifications and the weight of each brain region in the classification analysis were obtained. The brain regions with voxels that have values $\geq 30 \%$ of the maximum weight vector value of the discrimination map were taken as the key areas.

\section{Statistical Analysis}

The two-sample $t$ tests were employed to test the group differences in demographic data and imaging measures. Testing differences in FA values of the CST between the affected and unaffected sides were accomplished with a paired $t$ test. Correlations among the FA in key areas derived from MVPA and the CST were computed using Pearson's correlation coefficients. These statistical tests were performed using the Statistical Package for Social Science (SPSS) 22.0 software. The two-sample $t$ test was adopted for the inter-nodal connection comparisons followed by the NBS method using GRETNA (v2.0.0) to analyze the FA networks between groups. Visualization of the results was performed by the BrainNet Viewer $[26,27]$. The comparisons of the two sets of FA values in the key areas derived from MVPA were performed in SPM12 with false discovery rate (FDR)-corrected $\left(P_{F D R}<0.05\right.$, cluster size $\left.>5\right)$ [28].

\section{Results}

\section{Demographic data and Patient characteristics}

The demographic data of the patients and the controls are presented in Table 1. No significant differences in age or gender were observed between the two groups. The mean interval from stroke onset to DTI scans was $10.3 \pm 9.0$ weeks (Table 2 ). The lesions were mainly located in the basal ganglia region. For the 3 patients with lesions in the right hemisphere, the imaging data were laterally flipped. Stroke lesions for each patient were overlaid onto a T1 template in MNI standard space (Fig. 1).

The FA values of the ipsilesional CST in the patients were statistically significant reduced than that of the contralateral side ( $t=5.257, P=0.001)$. Compared with the controls, the FA values of the left (injured side) CST in the stroke group were significantly decreased $(t=6.511, P<0.001)$. And no significant differences in the FA values of the right CST $(t=0.153, P=0.88)$ were observed between the two groups.

\section{Overall Classifier Performance}

Figure $2 \mathrm{~A}$ shows the result of the MVPA classification between the 10 stroke patients and the 11 controls based on FA values. The overall accuracy was $100 \%$, and was significant at $P \leq 0.001(P=0.001)$. Both the sensitivity and the specificity were $100 \%$ (with ROC shown in Fig. 2B). This overall classification accuracy of the algorithm measures its ability to correctly sort the two groups. 
In the whole-brain voxel weight maps, the weight vector value indicates the relative importance of the voxel in the decision function -- discrimination between patients and controls (Fig. 3). Note that all voxels in the white matter mask contribute to the decision function since the analysis is multivariate. The spatial distribution of the weight vector provided information about the contribution of different areas to classification.

The brain regions that contributed the most to the discrimination between stroke patients and controls were identified by setting the threshold to $\geq 30 \%$ of the maximum weight vector scores, consistent with previous studies using MVPA for disease classification [11,29]. The most identified classified features of the FA maps included the perilesional basal ganglia and brainstem, with a few in the bilateral frontal lobes (Fig. 3 and 4 A), which were taken as the key areas.

By comparing the FA values of the two groups in the discrimination map by SPM12 $\left(P_{\mathrm{FDR}}<0.05\right.$, cluster size $>5$ ), we found that the stroke group showed reduced FA in the key areas, shown in Figure 4A and reported in Table 3. According to the white matter atlas, the clusters were mainly located in the CST pathway. Moreover, the FA values of these areas have a positive correlation with that of the ipsilesional CST $(r=0.888, p<0.001)$ (Fig. 4 B).

\section{Decreased Connection of the Component Network in stroke patients}

FA weighted networks were constructed from the nodes (brain areas) defined according to the AAL atlas. The weight of the edges in the network was defined as the mean FA value of the connected fibers between each pair nodes. The NBS approach was used on the structural networks constructed by deterministic tractography. We identified several significantly decreased connections of a component network (subnetwork) in stroke patients $(P<0.01, P=0.008)$ including 26 regions and 32 connections (Table 4 and Fig. 5 A).

Figure 5 showed the subnetwork that reveals deterioration in the brain structure of the stroke patients. Table 3 reported the regions as nodes in the subnetwork. The backbone matrix of the group structural matrixes for each group of the subnetwork was calculated, including the 26 regions in Table 4 (Fig. 5 B and $\mathrm{C}$ ). The subnetwork was composed of the cerebral cortical regions (the frontal lobe, the parietal lobe and the occipital lobe), the subcortical areas (the basal ganglion), and parts of the cerebellum. Compared with the results of MVPA, there were changes involving the frontal parietal lobe and the basal ganglia, and those in the brainstem region may overlap with some of the fibers connected to the cerebellum.

\section{Discussion}

Beyond the well-known concept of lesion-symptom mapping, some lesions in a single location in the brain could disrupt brain functions routed to widespread neural networks $[1,30]$. Our analysis confirmed that local destruction of certain anatomic regions could affect remote areas in the brain.

\section{White matter degeneration in the CST pathway of stroke patients}


The degree of anisotropy depends on the level of organization, the integrity of the white matter tract, and the degree of freedom of water diffusion movement caused by the oriented axonal membranes and myelin sheaths [31]. Reduced anisotropy along the CST far from the original lesions has been interpreted as Wallerian degeneration (WD) [32]. DTI can quantify the FA values to evaluate the pathology change of the white matter, such as WD. Using the good classification ability of MVPA, we reported that apart from the basal ganglia region which has direct infarcts, brain areas with significantly decreased FA values were also located in the brainstem of the lesioned hemisphere, and a few in the bilateral frontal lobes, which might be indicative of degenerative lesions caused by WD.

The acute and chronic phases of stroke probably differ in the white matter changes since neural changes can contain anterograde and retrograde degeneration, or refactoring. However, no matter what kind of alteration is present, it should generate specific structural changes and affect corresponding functions. Therefore we performed the pattern recognition classification using the whole brain FA map and explored the key brain regions important for distinguishing the stroke patients from the controls.

Our study verified degenerative changes in the white matter of stroke patients. The infarcted lesions were mainly located in the basal ganglia region, but the FA reduction of some remote areas has reached the point where they can be differentiated from the controls. In conclusion, the damaged structual anatomy of the subcortical areas may induce the deterioration of the key white matter areas in the brain.

\section{Decreased WM connections were widely distributed across brain regions of stroke patients}

Many patients were left with motor dysfunctions after the occurrence of cerebral infarction. Usually, it is related to the injury of the corticospinal tract. The CST originates from multiple motor and somatosensory cortices including premotor cortex, supplementary motor cortex (SMA), primary motor cortex, as well as primary and secondary somatosensory cortices. The CST is crucial for proper execution of a volitional movement $[33,34]$. Apart from the CST and the motor areas of the cortex, the proper execution of movements involving balance and coordination also requires the extrapyramidal tract and other brain regions such as the nucleus in basal ganglia and the cerebellum [35]. The NBS analysis showed that the structural subnetwork connection of the stroke group was weaker than that of the control group, which indicate that the nerve fibers involved in the subnetwork were affected. When the integrity and order of the brain structure were destroyed, it can be reflected in the white matter. Not only the brain regions directly related to the motor commands (e.g. precentral and SMA) but also other regions that might participate in the regulation of motor control were involved in the subnetwork.

In addition, since the MVPA analysis is multivariate, all voxels in the white matter mask contribute to the decision function in the processing stage. We observed that the area where the left CST passes is reddish, and there is a light green distribution in the corpus callosum and some other areas (Fig. 3). Combined with the NBS results, the affected brain tissues were distributed across a wide range of brain regions (Fig. 5). In short, the local abnormalities spread among the whole network as time elapses after the acute stage of the stroke onset. There is implicational information contained in the patterns of white matter degeneration. 
Next, we briefly discuss the brain regions involved in the subnetwork (Table 4).

\section{The bilateral frontal lobe}

Similar to previous studies results showing infarct-related focal thinning of the motor area in remote cortex via degeneration of inter-hemispheric connection fiber of the corpus callosum [36,37], we found changes in the connection between the frontal hemispheres, as well as reduced FA values in a small area located in the contralesional frontal cortex. It has been reported that secondary degeneration occurred in the ipsilesional precentral gyrus after subcortical stroke involving the CST at the 6-month follow-up in stroke patients by calculating the mean kurtosis (MK) value of manually drawing ROI from diffusion kurtosis imaging (DKI) imaging study [38]. We speculated that the degeneration in remote parts of the CST occurred in subacute phases of stroke.

In addition to motor-related brain areas, the affected brain areas in the frontal lobe may generate mild or long-term cognitive changes in patients. Recently, a research reported that the Reading the Mind in the Eyes Test (RMET) was associated with damages to the white-matter tracts connecting the frontal and the temporo-parietal components of the RMET functional network [39]. Cognitive impairment still requires sensitive detection of subtle changes by complex experimental design or long-term observations [40].

\section{The basal ganglia region}

The patients were infarcted mainly in the basal ganglia region. The CST section passing through the posterior limb of the internal capsule may receive regulatory information from the nucleus of basal ganglia or constitute loops within them. Due to the cortico-basal ganglia-thalamocortical 'motor' loop, any impact on the circuit constituent can lead to a shift in the balance between neural interactions in the direct and indirect pathways and subsequently lead to variations in the brain functions $[41,42]$. In the early stage of rehabilitation, stroke patients with hemiplegia often have synergistic movements, which are thought to be related to this loop.

\section{The occipital lobe}

In our study, changes in white matter connections involving the occipital (cuneus and precuneus) region were most likely related to visual effects. Voluntary actions modulate perception that follows the anatomical-functional bias of the motor system. Therefore stroke patients with dysfunction of normal voluntary movements may develop corresponding abnormal sensory modulations that gradually affect brain structure [43]. A study indicated a reduction in functional connectivity (FC) between the motor and executive control and the visuospatial networks in patients with motor deficits vs. healthy controls [44]. This demonstrated the existence of FC between the visual cortex, the ipsilateral/ contralateral motor cortex and the cerebellum. We conformed that this decline in FC is probably accompanied by a decrease in white matter structural connectivity.

\section{The cerebellum}


The cerebellum is often related to balance adjustment and patients with hemiplegia after stroke usually have problems of stability and coordination. The connections between the cerebrum and the cerebellum pass through the cerebral peduncle in the brainstem. So we assume that the weakening of those connections is partially consistent with the decrease in FA in the brainstem. Previous DTI studies also found decreased FA in midbrain of the stroke patients by manually plotting ROI [38]. A recent study indicated that the cerebellum plays a role on residual motor output by facilitating cortical excitability in chronic stroke [45]. Changes in cortico-cerebellar structural connectivity are probably caused by brain injuries related to the neural fibers connecting the cerebellum. The inability to perform normal movements might gradually lead to abnormal balance that is reflected in the decrease of cortico-cerebellar connectivity.

\section{Limitations and Expectations}

Despite our significant findings, this research is not free of limitations. First, the sample size is small. Second, the interval of DTI acquirement from stroke onset ranging from 2-24 weeks. Recovery of FA in penumbra regions occurs most rapidly during the first two weeks following stroke, with continued slow increases in FA for many weeks thereafter [46-47]. The phases of stroke rehabilitation may influence white matter organization considering that we only discussed stroke patients with motor impairments in a cross-sectional insight. Future work can be further explored by expanding the sample size and dynamically observing imaging changes from the acute phase to the recovery phase. Additionally, emphasis should be placed on the classification and refinement of clinical behaviors of stroke patients, with specific brain feature changes that correspond to the functional outcome.

Brain hemisphere dominance varies with different activities. Differences in the left and right hemisphere function often exist in emotion[48], language[49] and attention[50]. More studies are needed to fully understand all of the factors that affect this. Brain hemisphere dominance was little known in the motor movement. A study elucidated the symmetrical characteristics of the CST in bilateral hemispheres using probabilistic fiber tracking method. They clearly showed that the hand CST had symmetric structures in bilateral hemispheres[51]. When the researches emphasized different functions of the brain, especially the language, the two hemispheres are asymmetrical. In the future study, we should classify various functions of the brain and analyze the left or the right hemisphere separately.

A study on 132 stroke patients using rest-fMRI has revealed that although structural damage from stroke is focal, remote dysfunction can occur in regions of the brain distant from the lesions. These results link key organizational features of the brain networks to behavior in stroke [52]. To date, there is no largescale post-stroke structural network analysis similar in quantity and method to the above functional analysis. In this study, only stroke patients with motor dysfunction were recruited for analysis using DTI data. By MVPA and NBS, we detected the reduced FA values and abnormal white matter connections in the brain of those patients from a global level.

We need to further investigate the structure changes of the brain or the functional network in the natural process of different types of brain injuries. Then the diagnosis and prognosis can be more accurately 
assessed paving the way for better treatment selection and research methods.

\section{Conclusions}

In summary, we found that there are multiple WM structural abnormalities in the affected brain areas of stroke patients with motor impairment. Our study may provide a basis for further exploration of the neural mechanism involved in residual motor deficits in stroke patients.

Looking forward to the future, we will further compare the neural changes of well-recovered patients to provide a basis for the development of adaptive rehabilitation training strategies.

\section{Abbreviations}

DTI: Diffusion tensor imaging; MVPA: multivariate pattern analysis; NBS: network-based statistic; AAL: Automated Anatomical Labeling; FA: fractional anisotropy; MRI: Magnetic Resonance Imaging; SVM: support vector machine; WM: white matter; MD: mean diffusivity; RD: radial diffusivity; CST: corticospinal tract; ROI: region of interest ; MNI: Montreal Neurological Institute; FMA: Fugl-Meyer assessment; BRS: Brunnstrom stage; WD: Wallerian degeneration; DKI: Diffusion kurtosis imaging; RMET: Reading the Mind in the Eyes Test; FC: functional connectivity.

\section{Declarations}

\section{Availability of data and materials}

The data in the current study are available on reasonable request to the corresponding author.

\section{Ethics approval and consent to participate}

The study was approved by the local Ethics Committee of the Southeast University affiliated Zhongda Hospital. All participants gave written informed consent to participate according to the Declaration of Helsinki.

\section{Consent for publication}

Not applicable.

\section{Competing interests}

The authors declare that they have no competing interests

\section{Funding}

This work was funded by the National Natural Science Foundation of China (grant number:6590000127, 81801680), Nanjing health science and technology development special fund project (grant number: 
YKK18218).

\section{Acknowledgements}

We thank all the patients and volunteers for participating in this study.

\section{Authors' contributions}

XJ.C. and YJ.G. conceived and designed the research. XJ.C., Z.W, XH.C. and YL.L. performed the experiments and analyzed the data. X.Y. and W.W. provided technical assistance. SY.Z., XY.G. and SS.W. provided experimental assistance. XJ.C., IA.A. and YJ.G. wrote the manuscript.

\section{References}

1. Lim JS , Kang DW. Stroke connectome and its implications for cognitive and behavioral sequela of stroke. Journal of Stroke. J Stroke 2015;17:256-267. https://doi.org/10.5853/jos.2015.17.3.256

2. Cunningham DA, Machado A, Janini $D$, et al. Assessment of Inter-Hemispheric Imbalance Using Imaging and Noninvasive Brain Stimulation in Patients With Chronic Stroke. Arch Phys Med Rehabil 2015;96:S94-S103. https://doi.org/10.1016/j.apmr.2014.07.419

3. Visser MM, Nawaf Y, Campbell BCV, et al. White Matter Degeneration after Ischemic Stroke: A Longitudinal Diffusion Tensor Imaging Study. J Neuroimaging 2018;29:111-118. https://doi.org/10.1111/jon.12556

4. Byblow WD , Stinear CM , Barber PA, et al. Proportional recovery after stroke depends on corticomotor integrity. Ann Neurol 2015;78:848-859. https://doi.org/10.1002/ana.24472

5. Feng W, Wang J, Chhatbar PY, et al. Corticospinal tract lesion load - an imaging biomarker for stroke motor outcomes. Ann Neurol 2015;78:860-870. https://doi.org/10.1002/ana.24510

6. Meier EL, Johnson JP, Pan Y, et al. The utility of lesion classification in predicting language and treatment outcomes in chronic stroke-induced aphasia. Brain Imaging Behav 2019;13:1510. https://doi.org/10.1007/s11682-019-00118-3

7. Umarova RM , Beume $L$, Reisert $M$, et al. Distinct white matter alterations following severe stroke. Neurology 2017;88:1546-1555. https://doi.org/10.1212/WNL.0000000000003843

8. Jang SH, Kim K, Kim SH, et al. The relation between motor function of stroke patients and diffusion tensor imaging findings for the corticospinal tract. Neurosci Lett 2014;572:1-6. https://doi.org/10.1016/j.neulet.2014.04.044

9. Lao Z, Shen D, Xue Z, et al. Morphological classification of brains via high-dimensional shape transformations and machine learning methods. Neuroimage 2004;21:46-57. https://doi.org/10.1016/j.neuroimage.2003.09.027.

10. Pereira F, Mitchell T, Botvinick M. Machine learning classifiers and fMRI: A tutorial overview. Neurolmage 2009;45:S199-S209. https://doi.org/10.1016/j.neuroimage.2008.11.007 
11. Li F, Huang X, Tang W, et al. Multivariate pattern analysis of DTI reveals differential white matter in individuals with obsessive-compulsive disorder. Hum Brain Mapp 2014;35:2643-2651. https://doi.org/10.1002/hbm.22357

12. Janssen RJ, Mourão-Miranda J, Schnack HG. Making individual prognoses in psychiatry using neuroimaging and machine learning. Biol Psychiatr Cogn Neurosci Neuroimaging 2018;3:798-808. https://doi.org/10.1016/ j.bpsc.2018.04.004.

13. Zalesky A, Fornito A, Bullmore ET. Network-based statistic: Identifying differences in brain networks. Neurolmage 2010;53:1197-1207. https://doi.org/10.1016/j.neuroimage.2010.06.041

14. Fortanier E, Grapperon A-M, Le Troter A, et al. Structural connectivity alterations in amyotrophic lateral sclerosis: a graph theory based imaging study. Front Neurosci 2019;13:12. https://doi.org/10.3389/fnins.2019.01044.

15. Feng W, Wang J, Chhatbar PY, et al. Corticospinal tract lesion load: An imaging biomarker for stroke motor outcomes. Ann Neurol 2015;78:860-870. https://doi.org/10.1002/ana.24510

16. Naghdi S, Ansari NN, Mansouri K, et al. Neurophysiological and clinical study of Brunnstrom recovery stages in the upper limb following stroke. Brain Injury 2010;24:1372-1378. https://doi.org/10.3109/02699052.2010.506860

17. Grefkes C, Nowak DA, Eickhoff SB, et al. Cortical connectivity after subcortical stroke assessed with functional magnetic resonance imaging. Ann Neurol 2008;63:236-46. https://doi.org/10.1002/ana.21228

18. Chen JL, Schlaug G. Resting state interhemispheric motor connectivity and white matter integrity correlate with motor impairment in chronic stroke. Front Neurol 2013;4:178. https://doi.org/10.3389/fneur.2013.00178

19. Zhang $Y$, Liu $H$, Wang L, et al. Relationship between functional connectivity and motor function assessment in stroke patients with 26 hemiplegia: a resting-state functional MRI study. Neuroradiology 2016;58:503-511. https://doi.org/10.1007/ s00234- 016-1646-5.

20. Cui Z, Zhong $S$, Xu P, et al. PANDA: a pipeline toolbox for analyzing brain diffusion images. Front Hum Neurosci 2013;7:42. https://doi.org/10.3389/fnhum.2013.00042.

21. Guo X, Liu R, Lu J, et al. Alterations in brain structural connectivity after unilateral upper-limb amputation. IEEE Trans Neural Syst Rehabil Eng. 2019;27:2196-2204. https://doi.org/10.1109/TNSRE.2019.2936615.

22. Mori S, Crain BJ, Chacko VP, et al. Three-dimensional tracking of axonal projections in the brain by magnetic resonance imaging. Ann Neurol 1999;45:265-269. https://doi.org/10.1002/15318249(199902)45:2<265::AID-ANA21>3.0.C0;2-3

23. Tzourio-Mazoyer N, Landeau B, Papathanassiou D,et al. Automated anatomical labeling of activations in spm using a macroscopic anatomical parcellation of the mni mri single-subject brain. Neuroimage 2002;15:273-289. https://doi.org/10.1006/nimg.2001.0978

24. Ni S, Yaou L, Kuncheng L, et al. Diffusion tensor tractography reveals disrupted topological efficiency in white matter structural networks in multiple sclerosis. Cereb Cortex 2011;21:2565-2577. 
https://doi.org/10.1093/cercor/bhr039.

25. Schrouff J, Rosa MJ, Rondina JM, et al. PRoNTo: pattern recognition for neuroimaging toolbox. Neuroinformatics. 2013;11:319-337. https://doi.org/10.1007/s12021-013-9178-1.

26. Wang J, Wang $X$, Xia $M$, et al. GRETNA: a graph theoretical network analysis toolbox for imaging connectomics. Front Hum Neurosci 2015;9:386. https://doi.org/10.3389/fnhum.2015.00386.

27. Xia M, Wang J, He Y. BrainNet Viewer: a network visualization tool for human brain connectomics. Plos One 2013;8:e68910 https://doi.org/10.1371/journal.pone.0068910.

28. Liang $Y L$, Cai $X$, Zhou H,et al. Voxel-based analysis and multivariate pattern analysis of diffusion tensor imaging study in anti-NMDA receptor encephalitis. Neuroradiology 2020;62:231-239. https://doi.org/10.1007/s00234-019-02321-x

29. Ecker $C$, Rocha-Rego V, Johnston $P$, et al. Investigating the predictive value of whole-brain structural MR scans in autism: A pattern classification approach. Neurolmage 2010;49:44-56. https://doi.org/10.1016/j.neuroimage.2009.08.024.

30. Burke Quinlan E, Dodakian L, See J, et al. Neural function, injury, and stroke subtype predict treatment gains after stroke. Ann Neurol 2015;77:132-145. https://doi.org/10.1002/ana.24309

31. Virta A, Barnett A, Pierpaoli C. Visualizing and characterizing white matter fiber structure and architecture in the human pyramidal tract using diffusion tensor MRI. Magn Reson Imaging 1999;17:1121-1133. https://doi.org/10.1016/s0730-725x(99)00048-x

32. Thomalla G , Glauche V , Koch MA , et al. Diffusion tensor imaging detects early Wallerian degeneration of the pyramidal tract after ischemic stroke. Neurolmage 2004; 22:1767-1774. https://doi.org/10.1016/j.neuroimage.2004.03.041

33. Lemon RN. Descending pathways in motor control. Annu Rev Neurosci 2008;31:195-218. https://doi.org/10.1146/annurev.neuro.31.060407.125547.

34. Chenot Q, Tzourio-Mazoyer N, Rheault F, et al. A population-based atlas of the human pyramidal tract in 410 healthy participants. Brain Struct Funct 2019;224:599-612. https://doi.org/10.1007/s00429018-1798-7

35. Moreno-López Y, Olivares-Moreno R, Cordero-Erausquin M, et al. Sensorimotor integration by corticospinal system. Front Neuroanat 2016;10. https://doi.org/10.3389/fnana.2016.00024

36. Duering $M$, Righart $R$, Wollenweber FA, et al. Acute infarcts cause focal thinning in remote cortex via degeneration of connecting fiber tracts. Neurology 2015;84:1685-1692. https://doi.org/10.1212/WNL.0000000000001502

37. Hayward KS, Neva JL, Mang CS, et al. Interhemispheric pathways are important for motor outcome in individuals with chronic and severe upper limb impairment post stroke. Neural Plast 2017;2017:112. https://doi.org/10.1155/2017/4281532

38. Wei XE, Shang K, Zhou J, et al. Acute subcortical infarcts cause secondary degeneration in the remote non-involved cortex and connecting fiber tracts. Front Neurol 2019;10:9. https://doi.org/10.3389/fneur.2019.00860. 
39. Dominguez JFD, Nott Z, Horne K, et al. Structural and functional brain correlates of theory of mind impairment post-stroke. Cortex 2019;121:427-442. https://doi.org/10.1016/j.cortex.2019.09.017

40. Lim JSN, Kim MU, Jang MK, et al. Cortical hubs and subcortical cholinergic pathways as neural substrates of poststroke dementia. Stroke 2014;45:1069-1076. https://doi.org/10.1161/STROKEAHA.113.004156

41. Alexander GE, Crutcher MD, DeLong MR. Basal ganglia-thalamocortical circuits: parallel substrates for motor, oculomotor, "prefrontal" and "limbic" functions. Prog Brain Res 1990;85:119. https://doi.org/10.1016/S0079-6123(08)62678-3

42. Silkis I. The cortico-basal ganglia-thalamocortical circuit with synaptic plasticity. II. Mechanism of synergistic modulation of thalamic activity via the direct and indirect pathways through the basal ganglia. Biosystems 2001;59:7-14. https://doi.org/10.1016/S0303-2647(00)00135-0

43. Buaron B, Reznik D, Gilron R, et al. Voluntary actions modulate perception and neural representation of action-consequences in a hand-dependent manner. Cereb Cortex 2020. [epub] https://doi.org/10.1093/cercor/bhaa156..

44. Almeida SRM, Vicentini J, Bonilha L, et al. Brain connectivity and functional recovery in patients with ischemic stroke. J Neuroimaging 2017;27:65-70. https://doi.org/10.1111/jon.12362

45. Guder S, Frey BM, Backhaus W, et al. The Influence of Cortico-Cerebellar Structural Connectivity on Cortical Excitability in Chronic Stroke. Cereb Cortex 2020;30: 1330-1344. https://doi.org/10.1093/cercor/bhz169

46. Mandeville ET, Ayata $C$, Zheng $Y$, et al. Translational MR neuroimaging of stroke and recovery. Trans Stroke Res,2016;8:1-11. https://doi.org/10.1007/s12975-016-0497-z

47. Ding G, Jiang Q, Li L, et al. Magnetic resonance imaging investigation of axonal remodeling and angiogenesis after embolic stroke in sildenafil-treated rats. J Cereb Blood Flow Metab. 2008;28:1440-1448. https://doi.org/10.1038/jcbfm.2008.33

48. Lindell AK. Continuities in emotion lateralization in human and non-human primates. Front Hum Neurosci. 2013;7:464. https://doi.org/10.3389/fnhum.2013.00464

49. Hickok G, Poeppel D. The cortical organization of speech processing. Nat Rev Neurosci. 2007;8:393402. https://doi.org/10.1038/nrn2113.

50. Corballis, Michael C . Left brain, right brain: facts and fantasies. PLoS Biol. 2014;12:e1001767. https://doi.org/10.1371/journal.pbio.1001767

51. Lee DH, Lee DW, Han BS. Symmetrical location characteristics of corticospinal tract associated with hand movement in the human brain: A probabilistic diffusion tensor tractography. Medicine. 2016;95:e3317. https://doi.org/10.1097/MD.0000000000003317

52. Siegel JS, Ramsey LE, Snyder AZ, et al. Disruptions of network connectivity predict impairment in multiple behavioral domains after stroke. Proc Natl Acad Sci U. S. A. 2016;113:E4367-E4376. https://doi.org/10.1073/pnas.1521083113

\section{Tables}


Table 1. Demographic data of two groups.

\begin{tabular}{|llll|}
\hline & Control group & Stroke group & $P$ value \\
\hline Number & 11 & 10 & \\
\hline Gender(male/female) & $10 / 1$ & $7 / 3$ & 0.22 \\
\hline Age(mean \pm SD ,years) & $61.5 \pm 7.8$ & $56.7 \pm 10.5$ & 0.24 \\
\hline range(years) & $50-73$ & $35-74$ & \\
\hline
\end{tabular}

Table 2. Patient characteristics.

\begin{tabular}{|c|c|c|c|c|c|c|}
\hline ID & Age (years) & Side & Localization of infarct & BRS & FMA & $\begin{array}{l}\text { Scan time } \\
\text { (week) }\end{array}$ \\
\hline 1 & 49 & $\mathrm{R}$ & $B G$ & $3 \varangle 3 \rrbracket 5$ & 58 & 22 \\
\hline 2 & 67 & $\mathrm{R}$ & $B G$ & $2 \bigotimes 1 \rrbracket 4$ & 37 & 2 \\
\hline 3 & 57 & $\mathrm{~L}$ & $\mathrm{BG} \square \mathrm{PV}$ & $2 \bigotimes 1 \rrbracket 4$ & 35 & 4 \\
\hline 4 & 51 & $\mathrm{~L}$ & $\mathrm{BG} \square \mathrm{PV}$ & $3 \bowtie 2 \rrbracket 4$ & 41 & 10 \\
\hline 5 & 60 & $\mathrm{R}$ & $\mathrm{BG} \square \mathrm{CR}$ & $4 \llbracket 4 \rrbracket 5$ & 86 & 20 \\
\hline 6 & 57 & $\mathrm{~L}$ & $\mathrm{BG} \square \mathrm{CR}$ & $2 \otimes 1 \otimes 5$ & 38 & 3 \\
\hline 7 & 74 & $\mathrm{~L}$ & $\mathrm{BG} \square \mathrm{CR} \square \mathrm{PV}$ & $5 \llbracket 4 \rrbracket 5$ & 82 & 14 \\
\hline 8 & 60 & $\mathrm{~L}$ & $B G$ & $2 \bigotimes 1 \otimes 3$ & 19 & 2 \\
\hline 9 & 35 & $\mathrm{~L}$ & $B G$ & $2 \otimes 1 \otimes 3$ & 25 & 3 \\
\hline 10 & 57 & $\mathrm{~L}$ & $B G$ & $5 \rrbracket 5 \rrbracket 5$ & 89 & 24 \\
\hline
\end{tabular}

Side: the hemisphere of lesions on brain; $I C=$ internal capsule; $C R=$ corona radiate; $B G$ = basal ganglia; $\mathrm{PV}=$ periventricular ; $\mathrm{BRS}$ (Brunnstrom stage) : separate functional evaluation of proximal and distal portions of the upper and entire lower extremities. FMA: Fugl-Meyer assessment (full score=100). Scan time : intervel of DTI acquirement from stroke onset.

Table 3. Report of FA decreased brain areas in patients compared with controls. 


\begin{tabular}{|c|c|c|c|c|c|c|}
\hline ID & $\begin{array}{l}\text { Voxel } \\
\text { size }\end{array}$ & $\begin{array}{l}\text { Peak MNI } \\
\text { coordinate }(x, y, z)\end{array}$ & $\begin{array}{l}\text { Peak } \\
\text { intensity }\end{array}$ & Brain regions & $\begin{array}{l}\text { White matter } \\
\text { regions } \\
\text { (voxel size) }\end{array}$ & \\
\hline 1 & 36 & $-4-36-46$ & 0.028 & Pons; Medulla.L & CST.L & 20 \\
\hline \multirow[t]{2}{*}{2} & 114 & $\begin{array}{lll}-10 & -20 & -22\end{array}$ & 0.032 & Midbrain; Pons.L & CP.L & 57 \\
\hline & & & & & CST.L & 53 \\
\hline \multirow[t]{4}{*}{3} & 112 & $\begin{array}{lll}-22 & -6 & 16\end{array}$ & 0.027 & Extra-Nuclear; & PLIC.L & 43 \\
\hline & & & & Lentiform & SCR.L & 30 \\
\hline & & & & & SFOF.L & 15 \\
\hline & & & & & ALIC.L & 12 \\
\hline 4 & 9 & $-14 \quad-2060$ & 0.019 & MFG.L & $\begin{array}{l}\text { Not in the } \\
\text { atlas }\end{array}$ & \\
\hline 5 & 7 & $24-18 \quad 66$ & 0.023 & MFG.R & $\begin{array}{l}\text { Not in the } \\
\text { atlas }\end{array}$ & \\
\hline
\end{tabular}

ID : the index of the cluster ; Voxel size: the quantity of voxels in the cluster; Peak MNI coordinate: indicate the location of voxel with maximum weight vector scores (also peak intensity) in each cluster. Cluster Locater in PANDA software was used to locate the cluster image according to JHU ICBM-DTI-81 WhiteMatter Labels. White matter atlas (voxel size): the atlas regions this cluster involves and the quantity of voxels in this cluster overlapped with each atlas region. MFG: Medial Frontal Gyrus; CP:Cerebral peduncle ; PLIC: Posterior limb of internal capsule; SCR: Superior corona radiata ; SFOF:Superior fronto-occipital fasciculus (could be a part of anterior internal capsule) ; ALIC: Anterior limb of internal capsule; L:Left;R:Right.

Table 4. The 26 brain regions of the subnetwork nodes. 


\begin{tabular}{|c|c|c|c|c|c|c|}
\hline \multirow[t]{2}{*}{ ID } & \multirow[t]{2}{*}{ Region } & \multirow[t]{2}{*}{ Abbreviation } & \multirow[t]{2}{*}{ Side } & \multicolumn{3}{|c|}{ MNI coordinate } \\
\hline & & & & $x$ & $\mathrm{y}$ & $\mathrm{z}$ \\
\hline 1 & Precentral & PreCG & $\mathrm{L}$ & -39 & -6 & 51 \\
\hline 2 & Dorsolateral Frontal Superior gyrus & SFGdor & L & -18 & 35 & 42 \\
\hline 3 & Orbital Frontal Superior & ORBsup & $\mathrm{L}$ & -17 & 47 & -13 \\
\hline 4 & Frontal Middle gyrus & MFG & $\mathrm{L}$ & -33 & 33 & 35 \\
\hline 5 & Orbital Frontal Middle & ORBmid & $\mathrm{L}$ & -31 & 50 & -10 \\
\hline 6 & Opercular Frontal Inferior & IFGoperc & $\mathrm{L}$ & -48 & 13 & 19 \\
\hline 7 & Supplementary Motor Area & SMA & $\mathrm{L}$ & -5 & 5 & 61 \\
\hline 8 & Supplementary Motor Area & SMA & $\mathrm{R}$ & 9 & 0 & 62 \\
\hline 9 & Frontal Superior Medial & SFGmed & L & -5 & 49 & 31 \\
\hline 10 & Cuneus & CUN & $\mathrm{L}$ & -6 & -80 & 27 \\
\hline 11 & Occipital Superior gyrus & SOG & L & -17 & -84 & 28 \\
\hline 12 & Occipital Middle gyrus & MOG & L & -32 & -81 & 16 \\
\hline 13 & Occipital Inferior gyrus & IOG & L & -36 & -78 & -8 \\
\hline 14 & Postcentral & PoCG & $\mathrm{L}$ & -42 & -23 & 49 \\
\hline 15 & Parietal Superior gyrus & SPG & L & -23 & -60 & 59 \\
\hline 16 & Parietal Inferior gyrus & $\mathrm{IPL}$ & $\mathrm{L}$ & -43 & -46 & 47 \\
\hline 17 & Precuneus & PCUN & $\mathrm{L}$ & -7 & -56 & 48 \\
\hline 18 & Paracentral Lobule & PCL & $\mathrm{L}$ & -8 & -25 & 70 \\
\hline 19 & Caudate & CAU & $\mathrm{R}$ & 15 & 12 & 9 \\
\hline 20 & Putamen & PUT & L & -24 & 4 & 2 \\
\hline 21 & Pallidum & PAL & L & -18 & 0 & 0 \\
\hline 22 & Thalamus & THA & L & -11 & -18 & 8 \\
\hline 23 & Cerebellum Crus2 & CRBLCrus2 & L & -29 & -73 & -38 \\
\hline 24 & Cerebellum 3 & CRBL3 & $\mathrm{L}$ & -9 & -37 & -19 \\
\hline 25 & Cerebellum 4_5 & CRBL45 & L & -15 & -43 & -17 \\
\hline 26 & Cerebellum 9 & CRBL9 & L & -11 & -49 & -46 \\
\hline
\end{tabular}




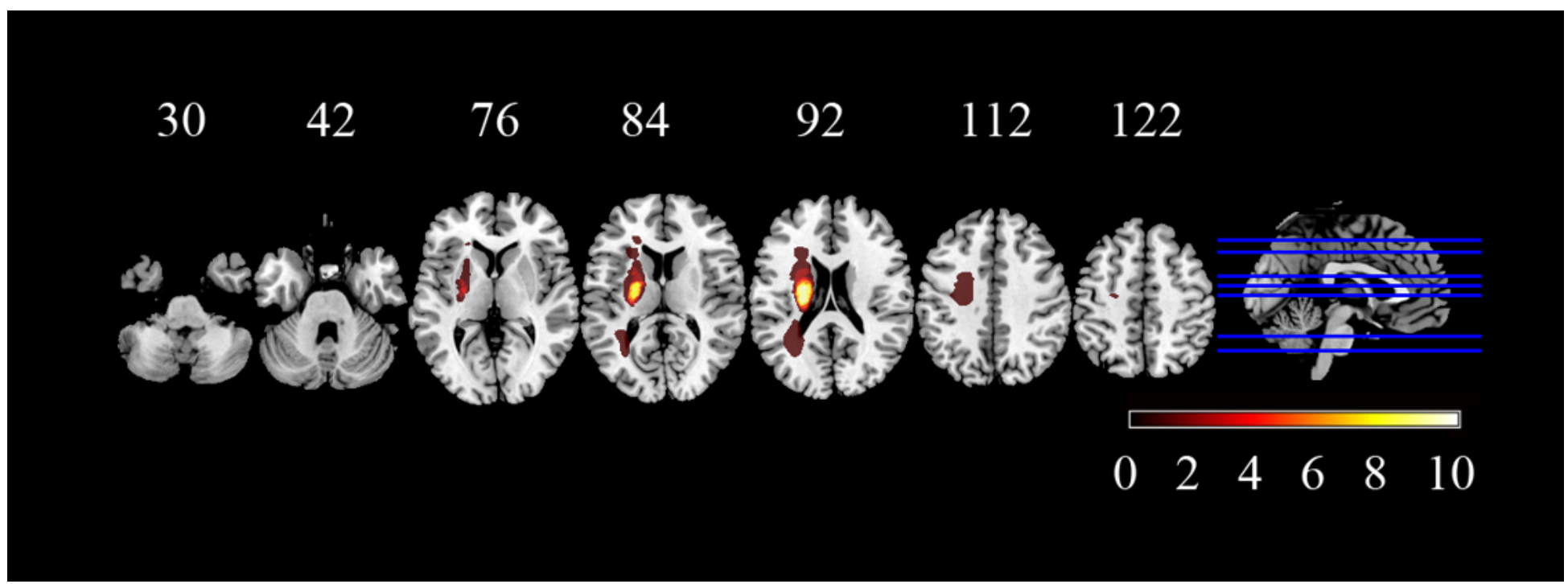

\section{Figure 1}

Lesion incidence map of patients with stroke. Stroke lesions were projected to the left hemisphere for each patient and overlaid onto a T1 template in MNI standard space. Colorbar indicates the number of patients with stroke lesions in the corresponding voxel. The numbers above the brain images are $Z$ values mark the MNI coordinates of the transverse sections.

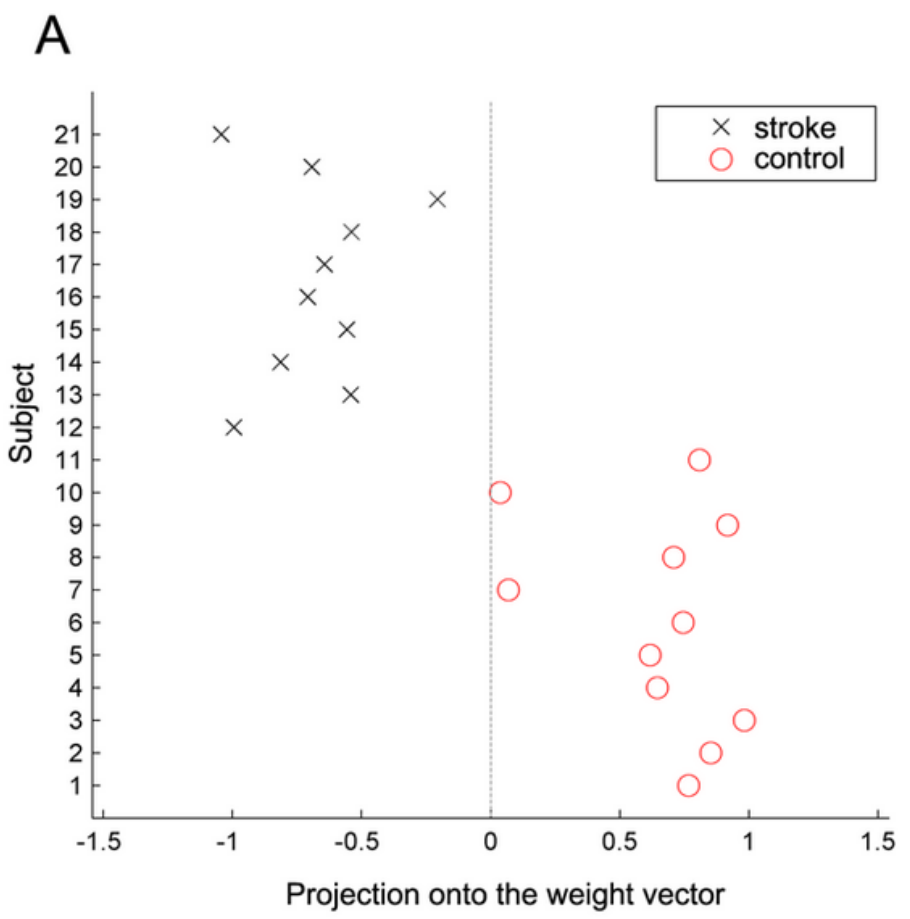

B Receiver Operator Curve/Area Under Curve $=1.00$

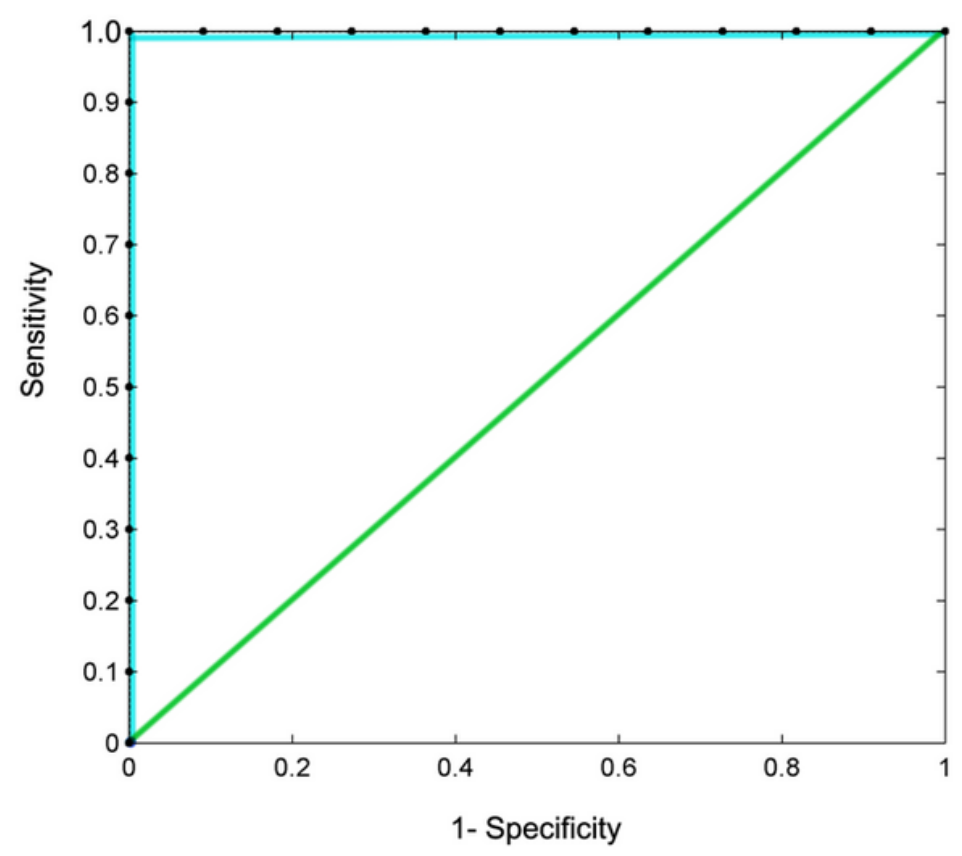

Figure 2 
The result of the MVPA classification. Classification plot (A) and Receiver Operating Characteristic (ROC) curve (B) for the comparison between stroke patients and controls using FA maps derived from DTI data, which yielded an accuracy of $100 \%$ (100\% sensitivity, $100 \%$ specificity), statistically significant at $\mathrm{P} \leq 0.001$.

\section{Wights map}
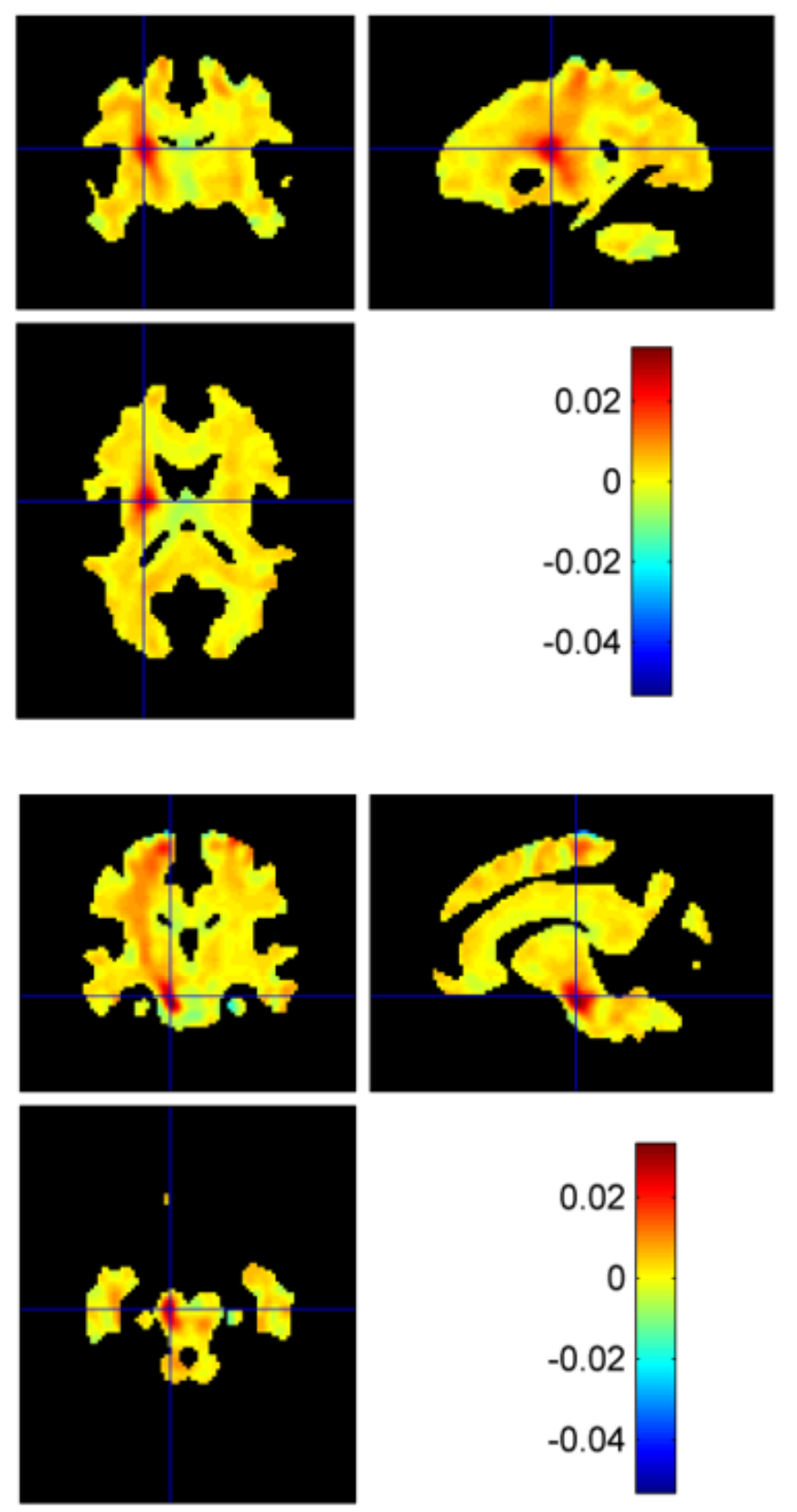
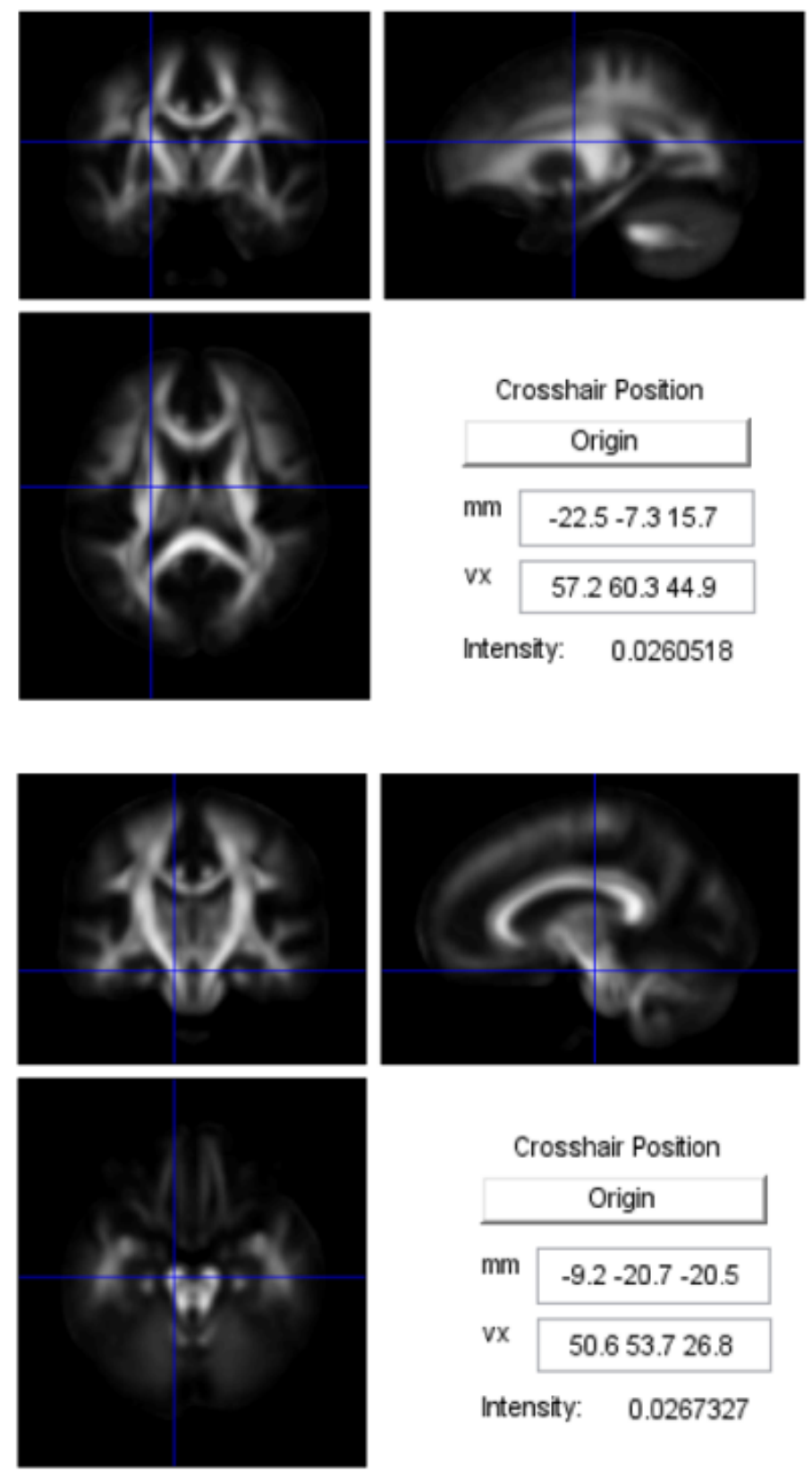

Crosshair Position

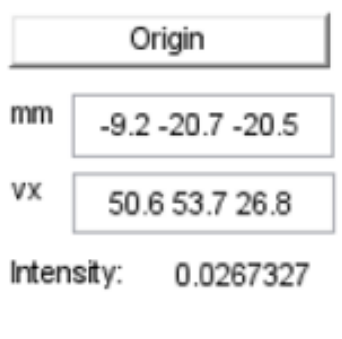

\section{Figure 3}

Whole-brain voxel weight map. It shows the white matter regions contributing to discrimination between groups based on FA values. The colour bar indicates the weight vector value of the voxel, which is also indicated in the intensity field of the Anatomical image (white fiber atlas 'JHU-ICBM-FA-2mm') panel. 
A
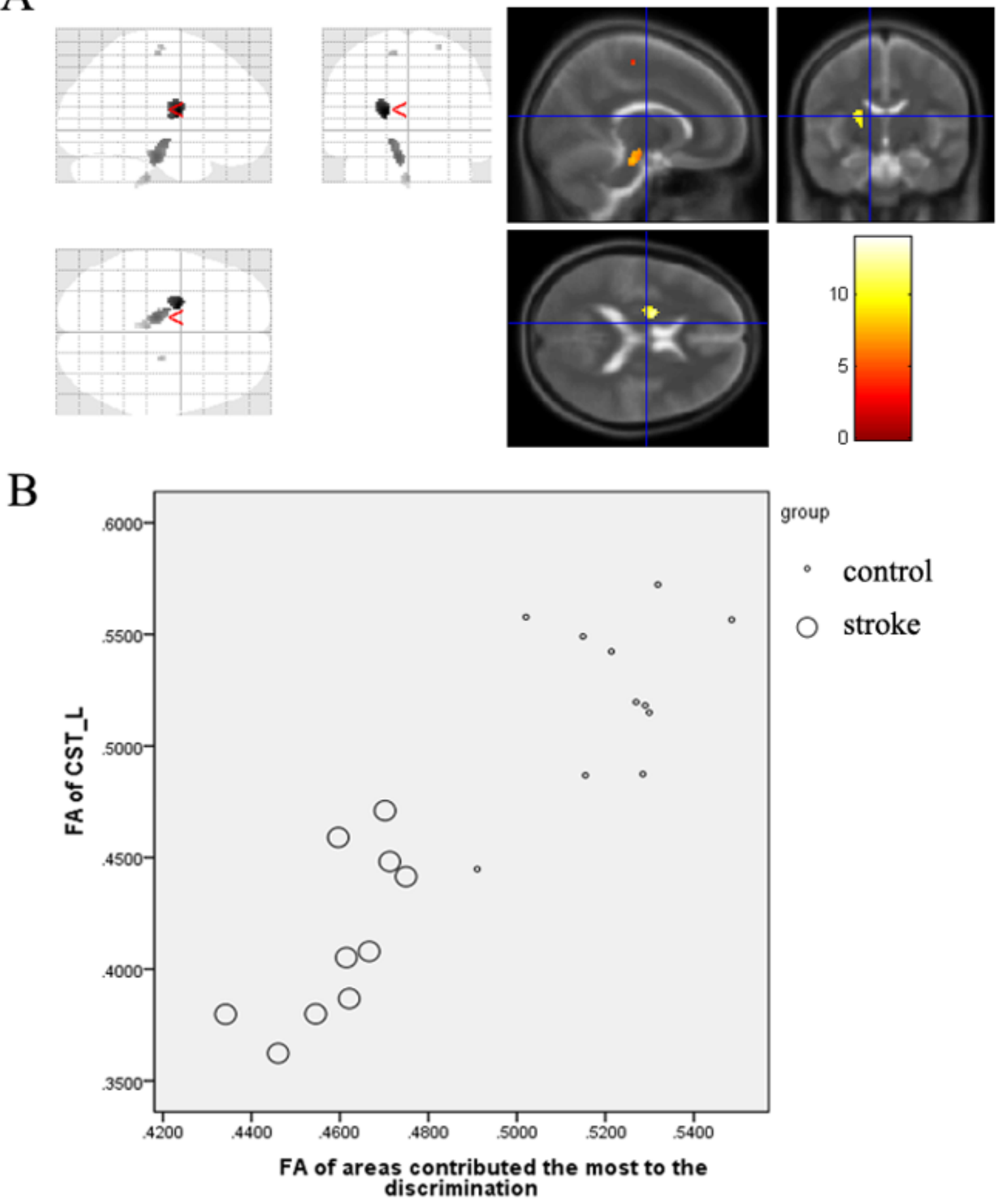

Figure 4

(A) The FA decreased brain areas in the patients compared to the controls including left brainstem, left basal ganglion and a fraction of voxels in bilateral parietal lobe. These regions were identified by setting the threshold to $\geq 30 \%$ of the maximum weight vector scores on the basis of the whole-brain voxel weight map. The colour bar indicates the T value in two-sample $t$ tests. (B) The FA value of the key areas have a positive correlation with that of the ipsilesional CST $(r=0.888, p<0.001)$. 
A

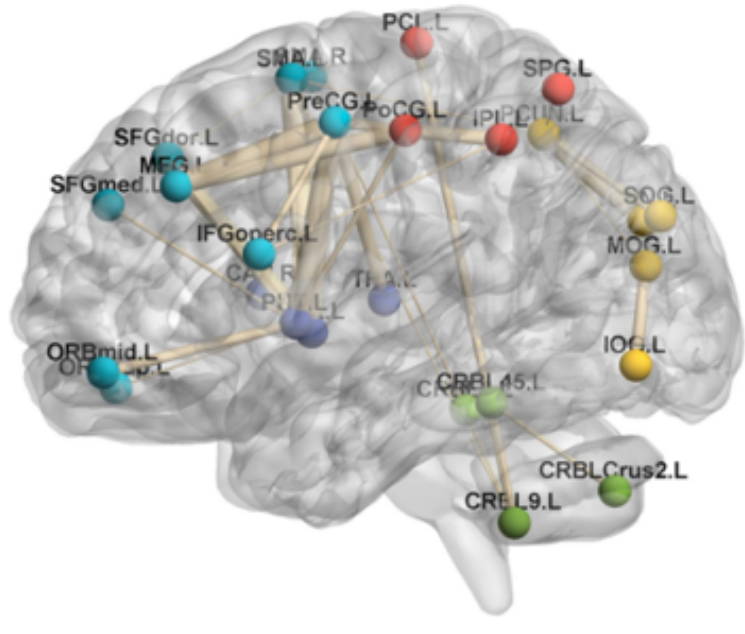

subcortical area

frontal lobe

cerebellum

occipital lobe

parietal lobe

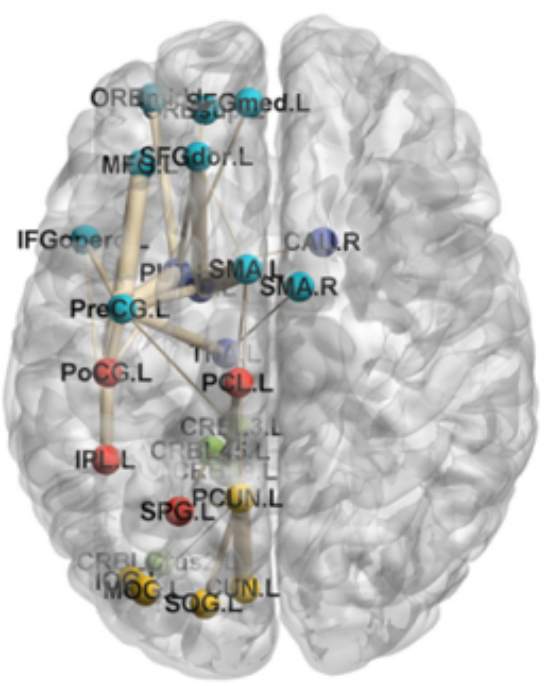

B
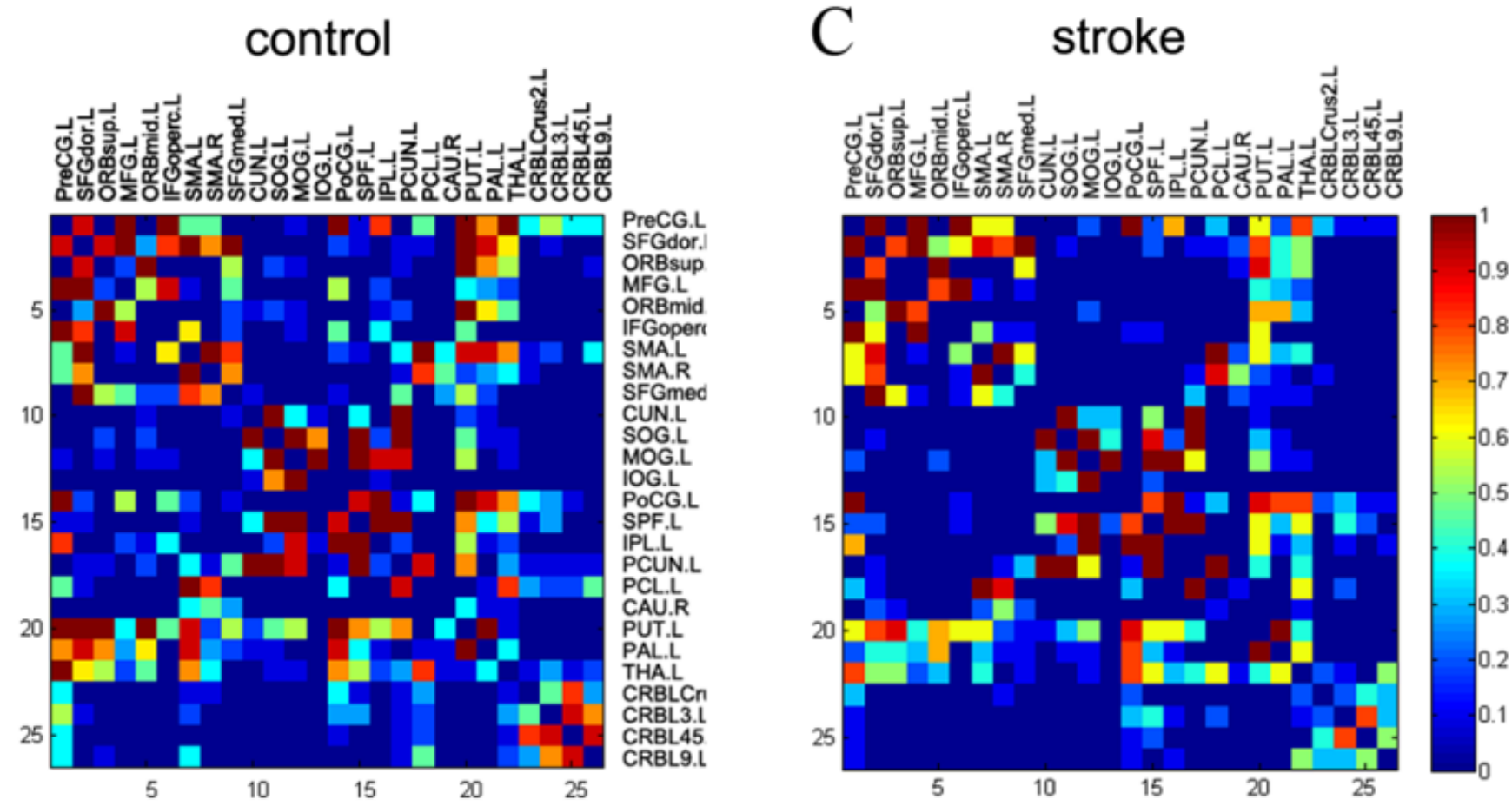

\section{Figure 5}

The subnetwork identified by the network-based statistics (NBS) analysis. (A) The subnetwork demonstrated reduced connectivity in stroke patients compared to controls ( the color circles represent different brain regions that were affected by the white matter disruption; thickness of edges represents how significantly 2 groups are different). The group averaged FA-weighted structural connectivity network for control group(B) and stroke group(C). The colorbar indicates connection probability in groups. 